\title{
Production of the $\Lambda_{c}(2940)$ by kaon-induced reactions on a proton target
}

\author{
Yin Huang, ${ }^{1}$ Jun He, ${ }^{2, *}$ Ju-Jun Xie, ${ }^{3}$ and Li-Sheng Geng ${ }^{1, \dagger}$ \\ ${ }^{1}$ School of Physics and Nuclear Energy Engineering, Beihang University, Beijing 100191, China \\ ${ }^{2}$ Department of Physics and Institute of Theoretical Physics, Nanjing Normal University, Nanjing 210097, \\ People's Republic of China \\ ${ }^{3}$ Institute of Modern Physics, Chinese Academy of Sciences, Lanzhou 730000, China
}

(Received 24 October 2016; revised manuscript received 30 November 2018; published 31 January 2019)

We investigate the possibility to study the charmed baryon $\Lambda_{c}(2940)$ by kaon-induced reactions on a proton target. Assuming that the $\Lambda_{c}(2940)$ is a $p D^{* 0}$ molecular state with spin parities $J^{P}=1 / 2^{ \pm}$, an effective Lagrangian approach is adopted to calculate the cross section and the $D^{0} p$ invariant mass spectrum of the $K^{-} p \rightarrow D_{s}^{-} \Lambda_{c}(2940)$ reaction. The $\bar{K} p$ initial state interaction mediated by Pomeron and Reggeon exchanges is also included, which reduces the production of the $\Lambda_{c}$ (2940). Besides, we also consider the $D N$ final state interaction using he chiral unitary approach. We found that the total cross section of the $K^{-} p \rightarrow$ $\Lambda_{c}(2940) D_{s}^{-}$reaction is about $10 \mu \mathrm{b}$. By considering the subsequential decay $\Lambda_{c}(2940) \rightarrow D^{0} p$ with contributions from the $\Lambda_{c}(2286)$ and the $\Sigma_{c}(2455)$ as background, the $K^{-} p \rightarrow D_{s}^{-} D^{0} p$ reaction is studied. It is found that the $\Lambda_{c}$ (2940) is produced mainly at forward angles. The $\Lambda_{c}(2940)$ signal is predicted to be significant in the $D^{0} p$ invariant mass spectrum of the $K^{-} p \rightarrow D_{s}^{-} D^{0} p$ reaction. The results suggest that it is promising to study the $\Lambda_{c}(2940)$ with high-energy kaon beams on a proton target.

DOI: $10.1103 /$ PhysRevD.99.014045

\section{INTRODUCTION}

In recent years, many charmed baryons have been observed. The charmed baryon $\Lambda_{c}(2940)$ was first observed by the $B A B A R$ Collaboration in the $D^{0} p$ invariant mass spectrum [1]. Later, the Belle collaboration also reported the observation of the $\Lambda_{c}(2940)$ in the $\Sigma_{c}(2455) \pi$ invariant mass spectrum [2]. The mass and width of the $\Lambda_{c}(2940)$ state reported by the above collaborations [1,2] are consistent with each other, i.e.,

$$
\begin{aligned}
\text { BABAR: } \mathrm{M} & =2939.8 \pm 1.3 \pm 1.0 \mathrm{MeV}, \\
\Gamma & =17.5 \pm 5.2 \pm 5.9 \mathrm{MeV} ; \\
\text { Belle: } \mathrm{M} & =2938.0 \pm 1.3_{-4.0}^{+2.0} \mathrm{MeV}, \\
\Gamma & =13_{-5-7}^{+8+27} \mathrm{MeV} .
\end{aligned}
$$

Since the mass of the $\Lambda_{c}(2940)$ is just a few $\mathrm{MeV}$ below the $D^{* 0} p$ threshold, it was proposed that this state is an $S$-wave $D^{* 0} p$ molecular state with a spin parity $J^{P}=1 / 2^{-}$,

\footnotetext{
* corresponding author; junhe@njnu.edu.cn

tlisheng.geng@buaa.edu.cn

Published by the American Physical Society under the terms of the Creative Commons Attribution 4.0 International license. Further distribution of this work must maintain attribution to the author(s) and the published article's title, journal citation, and DOI. Funded by SCOAP .
}

and the obtained decay behavior of $\Lambda_{c}(2940)$ is consistent with the experimental data [3]. Later, a study of the strong and radiative decays of the $\Lambda_{c}(2940)$ was performed by Dong and his collaborators $[4,5]$. Their results indicate that the $\Lambda_{c}(2940)$ can be viewed as a $D^{* 0} p$ molecular state with spin-parity $J^{P}=1 / 2^{+}$. In Ref. [6], a dynamical study of the $D^{* 0} p$ interaction in the one-boson-exchange model found two bound state solutions with quantum numbers $I\left(J^{P}\right)=$ $0\left(1 / 2^{+}\right)$and $0\left(3 / 2^{-}\right)$, which correspond to an isoscalar $S$-wave and an isoscalar $P$-wave $D^{* 0} p$ molecular state, respectively. In addition to the interpretation of the $\Lambda_{c}(2940)$ as a $p D^{* 0}$ molecular state, the possibility to assign it as a conventional charmed baryon was also discussed in many approaches, such as the potential model [7], the chiral perturbation theory [8], the ${ }^{3} P_{0}$ model [9], the relativistic quark-diquark model [10], the chiral quark model [11], the Faddeev method [12], and the mass load flux tube model [13].

The present knowledge about the $\Lambda_{c}(2940)$ was obtained from the $e^{+} e^{-}$collision [1,2]. Thus, it will be helpful to understand the nature of the $\Lambda_{c}(2940)$ if we can observe it in other production processes. In Refs. [14,15], a proposal was made to study the $\Lambda_{c}(2940)$ in the $\bar{p} p$ annihilation which can be performed in the future PANDA detector at FAIR. The production of the $\Lambda_{c}(2940)$ via a pion-induced reaction on a nucleon target was discussed in Ref. [16]. A study of the $\Lambda_{c}(2940)$ with electromagnetic probes in the $\gamma n \rightarrow \Lambda_{c}(2940) D^{-}$reaction was also proposed [17]. 
High-energy kaon beams are available at OKA@U-70 [18] and SPS@CERN [19], which provide another alternative to study charmed baryons. The kaon beam at J-PARC can also be upgraded to the energy region required in charmed baryon productions [20]. It is interesting to make a theoretical prediction about charmed baryon productions with kaon beams. With charged kaon beams, the $\Lambda_{c}(2940)$ can be produced with a proton target, e.g., the $K^{-} p \rightarrow D_{s}^{-} \Lambda_{c}(2940)$ reaction. In such a reaction, the $s$ channel is usually suppressed seriously because of the very large total energy $[16,21,22]$. The $u$-channel contribution is usually suppressed also and is more important at backward angles while the $\Lambda_{c}(2940)$ is produced at forward angles through the $t$ channel [23]. Moreover, compared with the pion-induced $\Lambda_{c}(2940)$ production, an additional $s \bar{s}$ quark pair creation is needed in the kaon-induced production, so the $u$ channel will be further suppressed. Hence, the $K^{-} p \rightarrow D_{s}^{-} \Lambda_{c}(2940)$ reaction should be dominant with the Born term through the $t$-channel $D^{* 0}$ exchange, which makes the background very small.

In the kaon-induced process considered in the current work, the effect from the $\bar{K} N$ initial state interaction (ISI) should be taken into account in order to make a more reliable prediction. Fortunately, there is plenty of experimental information about the $K^{-} p$ interaction in the energy region relevant to the $\Lambda_{c}(2940)$ production [24-27]. The existing experimental data show that the cross section of the $K^{-} p$ scattering is of the order of 10 millibarns in the energy range relevant here, that is, 1000 times larger than the theoretical predictions about the $\Lambda_{c}(2940)$ production in Refs. $[14,16,17]$, which are of the order of microbarns. Besides, recent theoretical studies also suggested that the interaction of a $D$ meson with a nucleon is of the same order of magnitude as that of the $K N$ interaction, i.e., tens of millibarns $[28,29]$. Hence, the $D N$ final state interaction (FSI) should be considered in the subsequential decay of the produced $\Lambda_{c}(2940)$.

In this work, we will study the $\Lambda_{c}(2940)$ production in the kaon-induced reaction in an effective Lagrangian approach with the ISI and FSI effects taken into account. The invariant mass spectrum for the subsequential decay of the $\Lambda_{c}(2940)$ in the $K^{-} p \rightarrow D_{s}^{-} \Lambda_{c}(2940) \rightarrow D_{s}^{-}\left(D^{0} p\right)$ reaction will be investigated as well.

This paper is organized as follows. In Sec. II, we will present the theoretical formalism. In Sec. III, the numerical result of the kaon-induced $\Lambda_{c}(2940)$ (we abbreviate it as $\Lambda_{c}^{*}$ hereafter) production on a proton target will be given. In Sec. IV, the invariant mass spectrum of the $K^{-} p \rightarrow D_{s}^{-} D^{0} p$ reaction will be presented, followed by discussions and conclusions in the last section.

\section{THEORETICAL FORMALISM}

In Fig. 1, we illustrate the Feynman diagram for the $t$-channel $K^{-} p \rightarrow D_{s}^{-} \Lambda_{c}^{*}$ interaction, which is the dominant

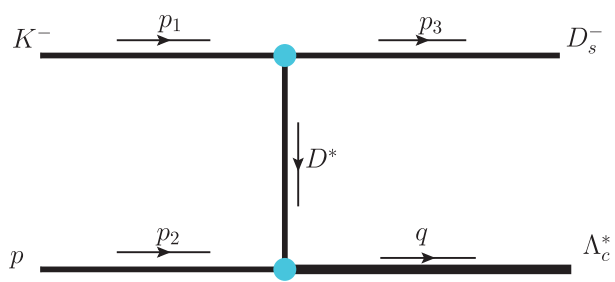

FIG. 1. Feynman diagram for the mechanism of the $\Lambda_{c}^{*+}$ production in the $K^{-} p \rightarrow D_{s}^{-} \Lambda_{c}^{*+}$ reaction. We also show the definition of the kinematics $\left(p_{1}, p_{2}, p_{3}\right.$, and $\left.q\right)$ used in the calculation.

mechanism of the $\Lambda_{c}^{*}$ production. The $\Lambda_{c}^{*}$ is produced by the exchange of a $D^{*}$ meson. As discussed in the Introduction, other production mechanisms will be suppressed heavily and not considered in this work.

To observe the $\Lambda_{c}^{*}$ in experiments, the subsequential decay to $D^{0} p$ shown in Fig. 2 will also be considered. The $\Lambda_{c}(2286)^{+}$and $\Sigma_{c}(2455)^{+}$can also be produced from the $K^{-}$and proton interaction by exchanging a $D^{*}$ meson and decay to $D^{0} p$. Therefore, in this work, the $\Lambda_{c}(2286)^{+}$and $\Sigma_{c}(2455)^{+}$will be taken as the background of the $\Lambda_{c}^{*}$ production.

\section{A. Lagrangians}

To compute the amplitudes of the diagrams shown in Figs. 1 and 2, we need the effective Lagrangian densities for the relevant interaction vertices. The spin parity of the $\Lambda_{c}^{*}$ state was still not determined in experiments. The theoretical studies suggested that possible assignments of spin parity of the $\Lambda_{c}^{*}$ are $J^{P}=1 / 2^{+}$and $1 / 2^{-}[3-5,15]$. In this work, we will consider these two possibilities. For the $\Lambda_{c}^{*} p D$ and $\Lambda_{c}^{*} p D^{*}$ couplings, we take the Lagrangian densities as used in Ref. [15],

$$
\begin{gathered}
\mathcal{L}_{\Lambda_{c}^{*} p D}=i g_{\Lambda_{c}^{*} p D} \bar{\Lambda}_{c}^{*} \gamma_{5} p D^{0}+\text { H.c. } \\
\mathcal{L}_{\Lambda_{c}^{*} p D^{*}}=g_{\Lambda_{c}^{*} p D^{*}} \bar{\Lambda}_{c}^{*} \gamma^{\mu} p D_{\mu}^{* 0}+\text { H.c. }
\end{gathered}
$$

for the case $J^{P}=1 / 2^{+}$, and

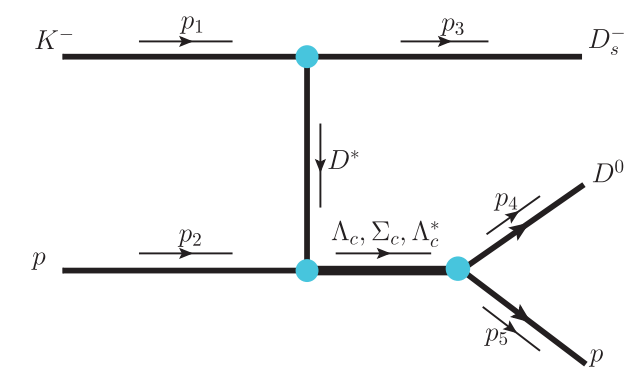

FIG. 2. Feynman diagram for the $K^{-} p \rightarrow D_{s} D^{0} p$ reaction. We also show the definition of the kinematics $\left(p_{1}, p_{2}, p_{3}, p_{4}\right.$, and $\left.p_{5}\right)$ used in the calculation. 


$$
\begin{gathered}
\mathcal{L}_{\Lambda_{c}^{*} p D}=g_{\Lambda_{c}^{*} p D} \bar{\Lambda}_{c}^{*} p D^{0}+\text { H.c. } \\
\mathcal{L}_{\Lambda_{c}^{*} p D^{*}}=-g_{\Lambda_{c}^{*} p D^{*}} \bar{\Lambda}_{c}^{*} \gamma_{5} \gamma^{\mu} p D_{\mu}^{* 0}+\text { H.c. }
\end{gathered}
$$

for the case $J^{P}=1 / 2^{-}$. The coupling constants in the above Lagrangians were determined in Refs. $[4,5]$ in a hadronic molecular picture with $g_{\Lambda_{c}^{*} p D}=-0.54, g_{\Lambda_{c}^{*} p D^{*}}=$ 6.64 for $J^{P}=1 / 2^{-}$and $f_{\Lambda_{c}^{*} p D}=-0.97, f_{\Lambda_{c}^{*} p D^{*}}=3.75$ for $J^{P}=1 / 2^{+}$.

For the $\Lambda_{c} p D, \Lambda_{c} p D^{*}, K D_{s} D^{*}, \Sigma_{c} N D$, and $\Sigma_{c} N D^{*}$ vertices, we adopt the commonly employed Lagrangian densities as follows [15,30,31],

$$
\begin{gathered}
\mathcal{L}_{\Lambda_{c} p D}=i g_{\Lambda_{c} p D} \bar{\Lambda}_{c} \gamma_{5} p D^{0}+\text { H.c., } \\
\mathcal{L}_{\Lambda_{c} p D^{*}}=g_{\Lambda_{c} p D^{*}} \bar{\Lambda}_{c} \gamma^{\mu} p D_{\mu}^{* 0}+\text { H.c. } \\
\mathcal{L}_{K D_{s} D^{*}}=i g_{K D_{s} D^{*}} D^{* \mu}\left[\bar{D}_{s} \partial_{\mu} K-\left(\partial_{\mu} \bar{D}_{s}\right) K\right]+\text { H.c., } \\
\mathcal{L}_{\Sigma_{c} N D}=-i g_{\Sigma_{c} N D} \bar{N} \gamma_{5} \tau \cdot \Sigma_{c} D+\text { H.c., } \\
\mathcal{L}_{\Sigma_{c} N D^{*}}=g_{\Sigma_{c} N D^{*}} \bar{N} \gamma_{\mu} \tau \cdot \Sigma_{c} D^{* \mu}+\text { H.c.. }
\end{gathered}
$$

The coupling constants $g_{\Lambda_{c} p D}=-13.98$ and $g_{\Lambda_{c} p D^{*}}=$ -5.20 are determined from the $\mathrm{SU}(4)$ invariant Lagrangians [5] in terms of $g_{\pi N N}=13.45$ and $g_{\rho N N}=$ 6.0. The coupling constants $g_{\Sigma_{c} N D}=2.69$ and $g_{\Sigma_{c} N D^{*}}=$ 3.0 [5]. The coupling constant $g_{K D_{s} D^{*}}=5.0$ can also be determined using the SU(4) symmetry [30,32,33].

When evaluating the scattering amplitude of the $K^{-} p \rightarrow$ $D_{s}^{-} \Lambda_{c}^{*}$ reaction, we need to include form factors because hadrons are not pointlike particles. We adopt here a common scheme used in many previous works $[14,16]$,

$$
F_{D^{*}}\left(q_{D^{*}}^{2}, M_{e x}\right)=\frac{\Lambda_{D^{*}}^{2}-M_{D^{*}}^{2}}{\Lambda_{D^{*}}^{2}-q_{D^{*}}^{2}},
$$

for $t$-channel $D^{*}$ meson exchange, and the form factor employed in Ref. [34],

$$
F_{B}\left(q_{B}^{2}, M_{B}\right)=\frac{\Lambda_{B}^{4}}{\Lambda_{B}^{4}+\left(q_{B}^{2}-M_{B}^{2}\right)^{2}}
$$

for the exchanged baryon, $\Lambda_{c}^{*}, \Lambda_{c}(2286)^{+}$or $\Sigma_{c}(2455)^{+}$. Here the $q_{\left(D^{*}, B\right)}$ and $M_{\left(D^{*}, B\right)}$ are the four momentum and the mass of the exchanged $D^{*}$ meson (baryon), respectively. In this work, we choose $\Lambda_{D^{*}}=\Lambda_{B}=3 \mathrm{GeV}$ to minimize the number of free parameters. This value is chosen in accordance with Refs. [15,35], and was employed in Refs. $[14,16]$. A variation of the cutoff will be made to show the sensitivity of the results on the cutoff.

\section{B. ISI and FSI}

Following Ref. [36] the initial state interaction for the $K^{-} p \rightarrow K^{-} p$ reaction at high energies will be taken into account. The amplitude $\mathcal{T}_{K^{-} p \rightarrow K^{-} p}$ is written in terms of the Pomeron and Reggeon exchanges [36]

$$
\mathcal{T}_{K^{-} p \rightarrow K^{-} p}=A_{P}+A_{f_{2}}+A_{a_{2}}+A_{\omega}+A_{\rho} .
$$

When the center-of-mass energy $\sqrt{s}$ is large, the elastic $K^{-} p$ scattering amplitude is a sum of the following terms,

$$
A_{i}(s, t)=\eta_{i} s C_{i}^{K N}\left(\frac{s}{s_{0}}\right)^{\alpha_{i}(t)-1} \exp \left(\frac{B_{K N}^{i}}{2} t\right),
$$

where $i=P$ for Pomeron and $f_{2}, a_{2}, \omega$, and $\rho$ Reggeons. The energy scale $s_{0}=1 \mathrm{GeV}^{2}$. The coupling constants $C_{i}^{K N}$, the parameters of the Regge linear trajectories $\left[\alpha_{i}(t)=\alpha_{i}(0)+\alpha_{i}^{\prime} t\right]$, the signature factors $\left(\eta_{i}\right)$, and the $B_{K N}^{i}$ used in Ref. [36] provide a rather good description of the experimental data. The parameters determined in Ref. [36] are listed in Table I.

The final state $D N$ interaction can be described by the chiral unitary approach of Ref. [29]. We choose two channels, $D p$ and $\pi \Sigma_{c}$, to reproduce the position of the $\Lambda_{c}(2595)$ resonance in isospin zero. Then, the amplitude $\mathcal{T}_{D^{0} p \rightarrow D^{0} p}$ can be obtained. We note that the other channels considered in Refs. [37-39], such as $\eta \Lambda_{c}, K \Xi_{c}, K \Xi_{c}^{\prime}, D_{s} \Lambda$, and $\eta^{\prime} \Lambda_{c}$, play a negligible role in dynamically generating the $\Lambda_{c}(2595)$ state, and therefore are not considered here.

In the chiral unitary approach, the scattering amplitude in the $D p$ and $\pi \Sigma_{c}$ coupled channels is given by

$$
\mathcal{T}=[1-V G]^{-1} V
$$

where $V$ is the chiral potential, and $G$ is a diagonal matrix, whose elements are loop functions of $D N$ or $\pi \Sigma_{c}$, defined as

$G=i \int \frac{d^{4} q}{(2 \pi)^{4}} \frac{1}{q^{2}-m_{i}^{2}+i \epsilon} \frac{2 M_{i}}{(p-q)^{2}-M_{i}^{2}+i \epsilon}$,

where $m$ and $M$ are the masses of the $\pi$ or $D(i=2)$ mesons and the baryons $\Sigma_{c}$ or $N(i=1)$, respectively. In the above equation, $p$ is the total incident momentum of the

TABLE I. Parameters of Pomeron and Reggeon exchanges determined from elastic and total cross sections in Ref. [36].

\begin{tabular}{lcccc}
\hline \hline$i$ & $\eta_{i}$ & $\alpha_{i}(t)$ & $C_{i}^{K N}(\mathrm{mb})$ & $B_{i}^{K N}\left(\mathrm{GeV}^{-2}\right)$ \\
\hline$P$ & $i$ & $1.081+\left(0.25 \mathrm{GeV}^{-2}\right) \mathrm{t}$ & 11.82 & 2.5 \\
$f_{2}$ & $-0.861+i$ & $0.548+\left(0.93 \mathrm{GeV}^{-2}\right) \mathrm{t}$ & 15.67 & 2.0 \\
$\rho$ & $-1.162-i$ & $0.548+\left(0.93 \mathrm{GeV}^{-2}\right) \mathrm{t}$ & 2.05 & 2.0 \\
$\omega$ & $-1.162-i$ & $0.548+\left(0.93 \mathrm{GeV}^{-2}\right) \mathrm{t}$ & 7.055 & 2.0 \\
$a_{2}$ & $-0.861+i$ & $0.548+\left(0.93 \mathrm{GeV}^{-2}\right) \mathrm{t}$ & 1.585 & 2.0 \\
\hline \hline
\end{tabular}


external meson-baryon system. The loop function $G$ can be regularized with dimensional regularization in terms of a subtraction constant,

$$
\begin{aligned}
G_{i}= & \frac{2 M_{i}}{(4 \pi)^{2}}\left\{a_{i}(\mu)+\log \frac{m_{i}^{2}}{\mu^{2}}+\frac{M_{i}^{2}-m_{i}^{2}+s}{2 s} \log \frac{M_{i}^{2}}{m_{i}^{2}}\right. \\
& +\frac{Q_{i}(\sqrt{s})}{\sqrt{s}}\left[\log \left(s-\left(M_{i}^{2}-m_{i}^{2}\right)+2 \sqrt{s} Q_{i}(\sqrt{s})\right)\right. \\
& +\log \left(s+\left(M_{i}^{2}-m_{i}^{2}\right)+2 \sqrt{s} Q_{i}(\sqrt{s})\right) \\
& -\log \left(-s+\left(M_{i}^{2}-m_{i}^{2}\right)+2 \sqrt{s} Q_{i}(\sqrt{s})\right) \\
& \left.\left.-\log \left(-s-\left(M_{i}^{2}-m_{i}^{2}\right)+2 \sqrt{s} Q_{i}(\sqrt{s})\right)\right]\right\},
\end{aligned}
$$

where $s=E^{2}$ with $E$ being the energy of the system in the center of mass frame, $Q_{i}$ is the on shell momentum of the particles in a certain channel, $\mu$ is the regularization scale, and $a_{i}(\mu)$ is the subtraction constant. With $\mu=1000 \mathrm{MeV}$, $a_{i}(\mu)=-2.02$, and the potential $V$ in Ref. [29], a rather narrow state around $2596 \mathrm{MeV}$ is dynamically generated, which indicates that the $\Lambda_{c}(2595)$ might be a $D N$ bound state. The obtained scattering amplitude $\mathcal{T}_{D^{0} p \rightarrow D^{0} p}$ will be used to calculate the FSI in the subsequantial decay of the $\Lambda_{c}^{*}$ in the following section.

\section{KAON-INDUCED $\Lambda_{c}^{*}$ PRODUCTION ON PROTON TARGET}

First, we calculate the total cross section of the $K^{-} p \rightarrow$ $\Lambda_{c}^{*} D_{s}$ reaction. The corresponding unpolarized differential cross section reads

$$
\frac{d \sigma}{d \cos \theta}=\frac{M_{p} M_{\Lambda_{c}^{*}}}{16 \pi s} \frac{\left|\vec{p}_{3 c m}\right|}{\left|\vec{p}_{1 c m}\right|}\left(\frac{1}{2} \sum_{s_{c}, s_{2}}\left|\mathcal{M}^{1 / 2^{ \pm}}\right|^{2}\right),
$$

where $s=\left(p_{1}+p_{2}\right)^{2}, \theta$ is the scattering angle of the outgoing $D_{s}^{-}$meson relative to the beam direction, $\vec{p}_{1 \mathrm{~cm}}$ and $\vec{p}_{3 \mathrm{~cm}}$ are the $K^{-}$and $D_{s}^{-}$three momenta in the center of mass frame, $M_{p}$ and $M_{\Lambda_{c}^{*}}$ are the masses of the proton and $\Lambda_{c}^{*}$, respectively.

Taking the ISI of the $K^{-} p$ system into account, the full amplitude for the process $K^{-} p \rightarrow \Lambda_{c}^{*} D_{s}$ is a sum of the Born and ISI amplitudes. With the Lagrangians given in the previous section, the Born amplitude of the $K^{-}\left(p_{1}\right) p\left(p_{2}\right) \rightarrow$ $\Lambda_{c}^{*}(q) D_{s}\left(p_{3}\right)$ reaction can be obtained as,

$$
\begin{aligned}
\mathcal{M}_{B}^{1 / 2^{ \pm}}= & g_{\Lambda_{c}^{*} p D^{*}} \bar{u}\left(q, s_{c}\right) \Gamma^{\mu \pm} u\left(p_{2}, s_{2}\right) G_{D^{*}}^{\mu \nu}\left(q_{D^{*}}\right) \\
& \times g_{K D_{s} D^{*}}\left(p_{1}^{\nu}+p_{3}^{\nu}\right) F_{D^{*}}^{2}\left(q_{D^{*}}^{2}, M_{D^{*}}\right),
\end{aligned}
$$

where $\Gamma^{\mu \pm}=\left(\gamma^{\mu},-\gamma_{5} \gamma^{\mu}\right)$, and $\bar{u}\left(q, s_{c}\right)$ and $u\left(p_{2}, s_{2}\right)$ are the Dirac spinors with $s_{c}(q)$ and $s_{2}\left(p_{2}\right)$ being the spins (the four-momenta) of the outgoing $\Lambda_{c}^{*}$ and the initial proton, respectively.

Following the strategy of Ref. [36], the ISI amplitude can be written as

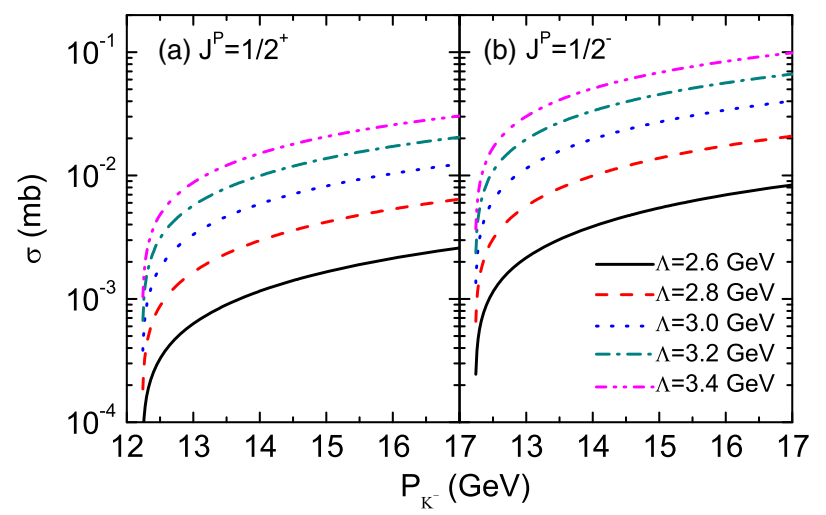

FIG. 3. Total cross section $\sigma$ for the $K^{-} p \rightarrow D_{s}^{-} \Lambda_{c}^{*}$ reaction as a function of the beam momentum $p_{K^{-}}$for the $J^{p}=1 / 2^{+}$case (left) and the $1 / 2^{-}$case (right).

$$
\begin{aligned}
\mathcal{M}_{I S I}^{1 / 2^{ \pm}}= & \frac{i}{16 \pi^{2} s} \int d^{2} \vec{k}_{t} \mathcal{T}_{K^{-} p \rightarrow K^{-} p}\left(s, k_{t}^{2}\right) \\
& \times \mathcal{M}_{B}^{1 / 2^{ \pm}}\left(-p_{2}-k_{t}+q\right),
\end{aligned}
$$

where $k_{t}$ is the momentum transfer in the $K^{-} p \rightarrow K^{-} p$ reaction.

In Fig. 3, the total cross section of the $K^{-} p \rightarrow D_{s}^{-} \Lambda_{c}^{*}$ reaction is shown as a function of the beam momentum. Because the cutoff can not be well determined, the results obtained with several cutoffs other than $3 \mathrm{GeV}$ are also presented.

The results show that the total cross section increases sharply near the $D_{s}^{-} \Lambda_{c}^{*}$ threshold. At higher energies, the cross section increases continuously but relatively slowly compared with that near threshold. With the increase of the cutoff, the total cross section increases, and generally speaking, the total cross section for $1 / 2^{+}$is smaller than that for $1 / 2^{-}$. At a beam momentum about $15 \mathrm{GeV}$ the cross section is of the order of $10 \mu \mathrm{b}$, which is quite large for an experimentally observation of the $\Lambda_{c}^{*}$ at current and future facilities.

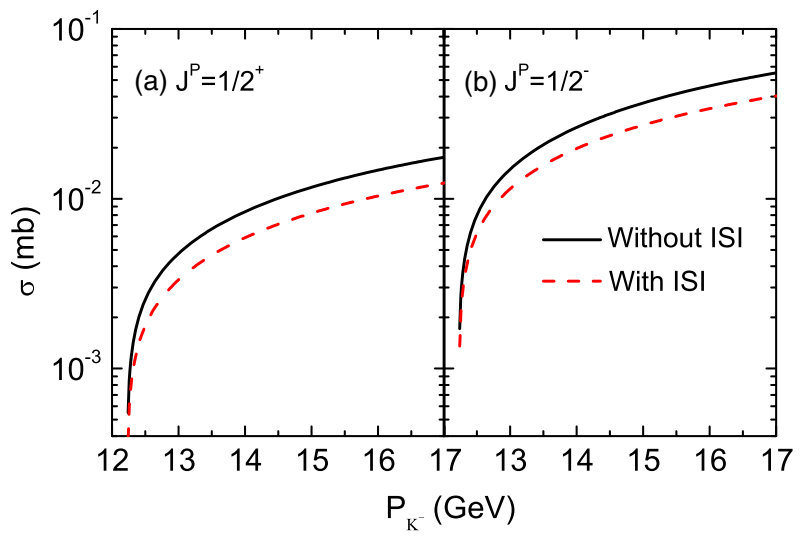

FIG. 4. Total cross section $\sigma$ with or without ISI for the $K^{-} p \rightarrow$ $D_{s}^{-} \Lambda_{c}^{*}$ reaction as a function of the beam momentum $p_{K^{-}}$for the $J^{p}=1 / 2^{+}$case (left) and the $1 / 2^{-}$case (right). 
As shown in Refs. [15,40,41], the ISI for $p p$ or $p \bar{p}$ reactions reduces the cross section. In Fig. 4 we compare the cross sections obtained with and without ISI for the cutoff of $\Lambda=3.0 \mathrm{GeV}$. It shows that the role of the ISI is to reduce the cross section by approximately $30 \%$.

\section{THE $K^{-} p \rightarrow D_{s}^{-} D^{0} p$ REACTION}

Since the $\Lambda_{c}^{*}$ can not be observed directly, the $D^{0} p$ channel of the $\Lambda_{c}^{*}$ decay, which is the observation channel of the $\Lambda_{c}^{*}$ at $B A B A R$, should be introduced to give a more realistic prediction for the observation of the $\Lambda_{c}^{*}$ in experiments. Here we consider the subsequential decay of the $\Lambda_{c}^{*}$ after being produced in the $K^{-} p \rightarrow \Lambda_{c}^{*} D_{s}^{-}$reaction, i.e., the $K^{-} p \rightarrow D_{s}^{-} D^{0} p$ reaction, which is illustrated in Fig. 2. The contributions from the $\Lambda_{c}(2286)$ and the $\Sigma_{c}(2455)$ will be included as background. Being a two-to-three-body process, the cross section can be obtained from the amplitude as,

$$
\begin{aligned}
d \sigma & \left(K^{-} p \rightarrow D_{s}^{-} D^{0} p\right) \\
= & \frac{M_{p}}{2 \sqrt{\left(p_{1} \cdot p_{2}\right)^{2}-M_{K^{-}}^{2} M_{p}^{2}}} \sum_{s_{i}, s_{f}}\left|\mathcal{M}\left(K^{-} p \rightarrow D_{s}^{-} D^{0} p\right)\right|^{2} \\
& \times \frac{d^{3} \vec{p}_{3}}{2 E_{3}} \frac{d^{3} \vec{p}_{4}}{2 E_{4}} \frac{M_{p} d^{3} \vec{p}_{5}}{E_{5}} \delta^{4}\left(p_{1}+p_{2}-p_{3}-p_{4}-p_{5}\right),
\end{aligned}
$$

where $M_{K^{-}}$is the mass of the kaon, and $E_{3}, E_{4}$, and $E_{5}$ stand for the energy of the $D_{s}^{-}, D^{-}$and final proton, respectively.

The amplitude $\mathcal{M}\left(K^{-} p \rightarrow D_{s}^{-} D^{0} p\right)$ can be obtained with the Lagrangians given in Sec. II as,

$$
\begin{aligned}
\mathcal{M}_{0}^{1 / 2^{+}}\left(K^{-} p \rightarrow D_{s}^{-} D^{0} p\right) & \\
= & i \frac{g_{\Lambda_{c}^{*} p D} g_{\Lambda_{c}^{*} p D^{*}} g_{K D_{s} D^{*}}}{q^{2}-M_{\Lambda_{c}^{*}}^{2}+i M_{\Lambda_{c}^{*}} \Gamma_{\Lambda_{c}^{*}}} \\
& \times \frac{1}{k^{2}-M_{D^{* 0}}^{2}} F_{\Lambda_{c}^{*}}\left(q^{2}, M_{\Lambda_{c}^{*}}\right) F_{D^{*}}^{2}\left(k^{2}, M_{D^{*}}\right)\left(p_{1 \mu}+p_{3 \mu}\right) \\
& \times \bar{u}\left(p_{5}, s_{5}\right) \gamma_{5}\left(\not q+M_{\Lambda_{c}^{*}}\right)\left(\gamma^{\mu}-\frac{\not k k^{\mu}}{M_{D^{*}}^{2}}\right) u\left(p_{2}, s_{2}\right),
\end{aligned}
$$

$$
\begin{aligned}
\mathcal{M}_{0}^{1 / 2^{-}} & \left(K^{-} p \rightarrow D_{s}^{-} D^{0} p\right) \\
= & \frac{f_{\Lambda_{c}^{*} p D} f_{\Lambda_{c}^{*} p D^{*}} g_{K D_{s} D^{*}}}{q^{2}-M_{\Lambda_{c}^{*}}^{2}+i M_{\Lambda_{c}^{*}} \Gamma_{\Lambda_{c}^{*}}} \\
& \times \frac{1}{k^{2}-M_{D^{* 0}}^{2}} F_{\Lambda_{c}^{*}}\left(q^{2}, M_{\Lambda_{c}^{*}}\right) F_{D^{*}}^{2}\left(k^{2}, M_{D^{*}}\right)\left(p_{1 \mu}+p_{3 \mu}\right) \\
& \times \bar{u}\left(p_{5}, s_{5}\right)\left(\not q+M_{\Lambda_{c}^{*}}\right) \gamma_{5}\left(\gamma^{\mu}-\frac{\not k k^{\mu}}{M_{D^{*}}^{2}}\right) u\left(p_{2}, s_{2}\right),
\end{aligned}
$$

and the amplitudes for the $\Lambda_{c}(2286)$ and the $\Sigma_{c}(2455)$ can be obtained analogously. Here we take $\Gamma=0 \mathrm{MeV}$ for the $\Lambda_{c}(2286)$ and the $\Sigma_{c}(2455)$ states because of their small decay widths. For the $\Lambda_{c}^{*}$, the width is taken to be $\Gamma=$ $17 \mathrm{MeV}$ according to $B A B A R$, consistent with the one obtained by Belle [42].

Taking the ISI of the $K^{-} p$ and FSI of the $D^{0} N$ systems into account, the amplitude of the $K^{-} p \rightarrow D_{s}^{-} D^{0} p$ reaction can be written as

$$
\begin{aligned}
\mathcal{M}^{1 / 2^{ \pm}}\left(K^{-} p \rightarrow D_{s}^{-} D^{0} p\right) \\
=\left[\mathcal{M}_{0}^{1 / 2^{ \pm}}(s)+\frac{i}{16 \pi^{2} s} \int d^{2} \vec{k}_{t}\right. \\
\left.\quad \times \mathcal{T}_{K^{-} p \rightarrow K^{-} p}\left(s, k_{t}^{2}\right) \mathcal{M}_{0}^{1 / 2^{ \pm}}\left(-p_{2}-k_{t}+q\right)\right] \\
\quad \times\left(1+G_{D^{0} p} \mathcal{T}_{D^{0} p \rightarrow D^{0} p}\right) .
\end{aligned}
$$

With the formalism and ingredients given above, the cross section as a function of the beam momentum $p_{K^{-}}$for the $K^{-} p \rightarrow D_{s}^{-} D^{0} p$ reaction is calculated using a Monte Carlo multiparticle phase space integration program, FOWL, and crosschecked with direct integration with Eq. (20). The theoretical results at a cutoff $\Lambda=3.0 \mathrm{GeV}$ for the beam momentum $p_{K^{-}}$from near threshold up to $16.5 \mathrm{GeV}$ are shown in Fig. 5. The contributions from the $\Lambda_{c}(2286)$ and the $\Lambda_{c}(2455)$ are also presented in the same figure.

As in the $\Lambda_{c}^{*}$ production, the total cross section of the $\Lambda_{c}^{*}$ for the $J^{P}=1 / 2^{-}$case is much larger than that for the $1 / 2^{+}$case. The order of magnitude of the total cross section is about 1 and $10 \mu \mathrm{b}$ for positive and negative parities, respectively. The background contribution from the $\Sigma_{c}(2455)$ to the total cross section is much smaller than all the other contributions. The contribution from the $\Lambda_{c}^{*}$ is much larger than all the other contributions if the spin

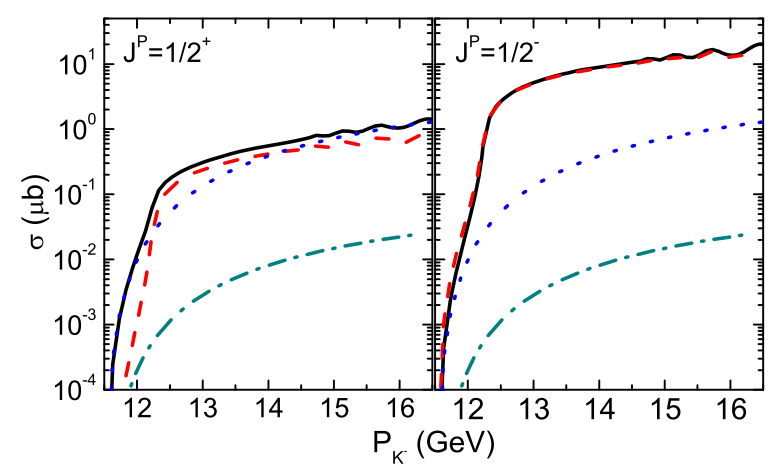

FIG. 5. Total cross section $\sigma$ for the $K^{-} p \rightarrow D_{s}^{-} D^{0} p$ reaction as a function of the beam momentum $p_{K^{-}}$for $J^{P}=1 / 2^{+}$(left) and $J^{P}=1 / 2^{-}$(right). The dashed, dotted, and dash-dotted curves stand for the contributions from the $\Lambda_{c}^{*}$, the $\Lambda_{c}(2286)$, and the $\Sigma_{c}(2455)$, respectively. The total contribution is shown by the solid line. 


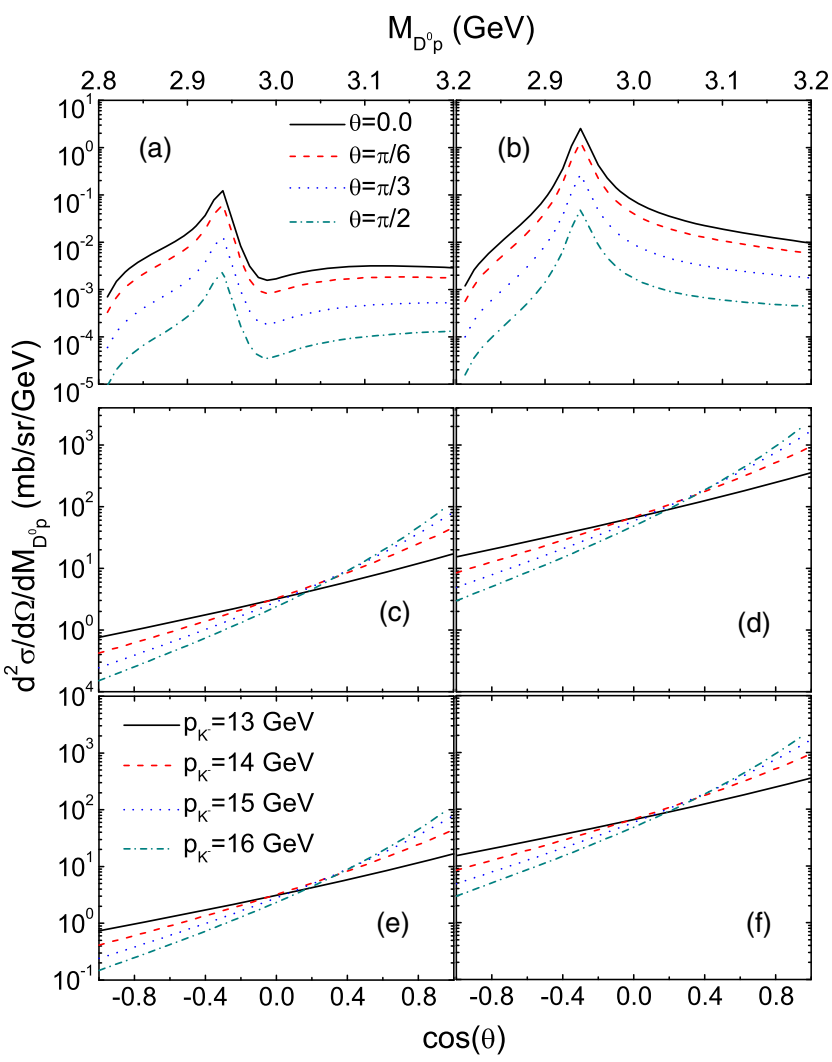

FIG. 6. Second order differential cross section $d^{2} \sigma / d \Omega / d M_{D^{0} p}$ for the $K^{-} p \rightarrow D_{s}^{-} D^{0} p$ reaction as a function of the $p D^{0}$ invariant mass $M_{D^{0} p}$ (a and b), and the scattering angle $\cos \theta$ (c, d, e, and f). The subfigures (a, c, and e) are for $J^{P}=1 / 2^{+}$ case, and the subfigures (b,d, and f) are for $1 / 2^{+}$. The subfigures (c and d) are for the cross section with background, and the subfigures (e and $\mathrm{f}$ ) are for the cross section without background.

parity of the $\Lambda_{c}^{*}$ is $1 / 2^{-}$, which makes the observation of the $\Lambda_{c}^{*}$ easy. However, if the $\Lambda_{c}^{*}$ carries a spin parity of $1 / 2^{+}$, the total cross section from the $\Lambda_{c}^{*}$ is much smaller and comparable with the background contribution especially that from the $\Lambda_{c}^{*}(2286)$.

To give more information about the $\Lambda_{c}^{*}$ production in the $K^{-} p \rightarrow D_{s}^{-} D^{0} p$ reaction, we present the second order differential cross section $d^{2} \sigma / d \Omega / d M_{D^{0} p}$ as a function of the $p D^{0}$ invariant mass for $p_{K^{-}}=16 \mathrm{GeV}$ at several typical scattering angles, $\theta=0, \pi / 6, \pi / 3$, and $\pi / 2$, in Figs. 6(a) and 6(b) for positive and negative parities of the $\Lambda_{c}^{*}$, respectively. An obvious peak can be found around the invariant mass $M_{D^{0} p}=2.94 \mathrm{GeV}$ as expected. The differential cross section is the largest at the extreme forward angle and decreases with the increase of the scattering angle. It can be seen more clearly at the differential cross sections as a function of the scattering angle at the invariant mass $M_{p D^{0}}=2.94 \mathrm{GeV}$ in Fig. 6(c)-6(f).

In Figs. 6(c) and 6(d), the results for the $K^{-} p \rightarrow D_{s}^{-} D^{0} p$ reaction at different kaon momenta $p_{K^{-}}$are presented. The results show that with the increase of the kaon momentum, the increase of the total cross section shown in Fig. 5 is mainly from the increase of the differential cross section at forward angles.

We also present the results of the $K^{-} p \rightarrow D_{s}^{-} D^{0} p$ reaction through $\Lambda_{c}^{*}$ only in Figs. 6(e) and 6(f), which shows the effects of the background on the differential cross sections as a function of the scattering angle is very small. With the increase of the kaon momentum, more $\Lambda(2940)$ can be produced at forward angles. The results suggest that it is better to observe the $\Lambda_{c}^{*}$ at forward angles especially at high energies.

\section{SUMMARY}

In this work, we studied the $\Lambda_{c}^{*}$ production in the $K^{-} p \rightarrow$ $D_{s}^{-} \Lambda_{c}^{*}$ and the $K^{-} p \rightarrow D_{s}^{-} D^{0} p$ reactions within the effective Lagrangian approach to investigate the possibility to produce the charmed baryon $\Lambda_{c}^{*}(2940)$ with kaon beams on a proton target. The $t$-channel $D^{*}$ exchange was considered to be the dominant production mechanism. The $\bar{K} N$ initial state interaction(ISI) was included by Pomeron and Reggeon exchanges [36], which was shown to reduce the cross section by about $30 \%$. In addition, we have also considered the $D N$ final state interaction (FSI) with the amplitudes provided by the chiral unitary approach [29].

The total cross section of the $\Lambda_{c}^{*}$ production was found to be at the order of $10 \mu \mathrm{b}$. After considering the subsequential decay of the $\Lambda_{c}^{*}$ in the $D^{0} p$ channel, we found a clear signal of the $\Lambda_{c}^{*}$ in the $D^{0} p$ invariant mass spectrum of the $K^{-} p \rightarrow D_{s}^{-} D^{0} p$ reaction. Our study indicates that it is feasible to produce the $\Lambda_{c}(2940)$ with kaon beams on a proton target.

\section{ACKNOWLEDGMENTS}

This project is partially supported by the National Natural Science Foundation of China (Grant No. 11675228, No. 11522539, No. 11735003, and No. 11475227), the Major State Basic Research Development Program in China (No. 2014CB845405). 
[1] B. Aubert et al. (BABAR Collaboration), Observation of a Charmed Baryon Decaying to D0p at a Mass Near 2.94-GeV/c², Phys. Rev. Lett. 98, 012001 (2007).

[2] K. Abe et al. (Belle Collaboration), Experimental Constraints on the Possible $J^{P}$ Quantum Numbers of the $\Lambda_{c}(2880)^{+}$, Phys. Rev. Lett. 98, 262001 (2007).

[3] X. G. He, X. Q. Li, X. Liu, and X. Q. Zeng, $\Lambda_{c}^{+}$(2940): A possible molecular state?, Eur. Phys. J. C 51, 883 (2007).

[4] Y. Dong, A. Faessler, T. Gutsche, and V. E. Lyubovitskij, Strong two-body decays of the $\Lambda_{c}(2940)^{+}$in a hadronic molecule picture, Phys. Rev. D 81, 014006 (2010).

[5] Y. Dong, A. Faessler, T. Gutsche, S. Kumano, and V. E. Lyubovitskij, Radiative decay of $\Lambda_{c}(2940)^{+}$in a hadronic molecule picture, Phys. Rev. D 82, 034035 (2010).

[6] J. He and X. Liu, The observed charmed hadron $\Lambda_{c}(2940)^{+}$ and the $D^{*} N$ interaction, Phys. Rev. D 82, 114029 (2010).

[7] S. Capstick and N. Isgur, Baryons in a relativized quark model with chromodynamics, Phys. Rev. D 34, 2809 (1986).

[8] H. Y. Cheng and C. K. Chua, Strong decays of charmed baryons in heavy hadron chiral perturbation theory, Phys. Rev. D 75, 014006 (2007).

[9] C. Chen, X. L. Chen, X. Liu, W. Z. Deng, and S. L. Zhu, Strong decays of charmed baryons, Phys. Rev. D 75, 094017 (2007).

[10] D. Ebert, R. N. Faustov, and V. O. Galkin, Masses of excited heavy baryons in the relativistic quark model, Phys. Lett. B 659, 612 (2008).

[11] X. H. Zhong and Q. Zhao, Charmed baryon strong decays in a chiral quark model, Phys. Rev. D 77, 074008 (2008).

[12] A. Valcarce, H. Garcilazo, and J. Vijande, Towards an understanding of heavy baryon spectroscopy, Eur. Phys. J. A 37, 217 (2008).

[13] B. Chen, D. X. Wang, and A. Zhang, $J^{P}$ Assignments of $\Lambda_{c}^{+}$ Baryons, Chin. Phys. C 33, 1327 (2009).

[14] J. He, Z. Ouyang, X. Liu, and X. Q. Li, Production of charmed baryon $\Lambda_{c}(2940)^{+}$at PANDA, Phys. Rev. D 84, 114010 (2011).

[15] Y. Dong, A. Faessler, T. Gutsche, and V. E. Lyubovitskij, Role of the hadron molecule $\Lambda_{c}(2940)$ in the $p \bar{p} \rightarrow$ $p D^{0} \bar{\Lambda}_{c}$ (2286) annihilation reaction, Phys. Rev. D 90, 094001 (2014).

[16] J. J. Xie, Y. B. Dong, and X. Cao, Role of the $\Lambda_{c}^{+}(2940)$ in the $\pi^{-} p \rightarrow D^{-} D^{0} p$ reaction close to threshold, Phys. Rev. D 92, 034029 (2015).

[17] X. Y. Wang, A. Guskov, and X. R. Chen, $\Lambda_{c}^{*}(2940)^{+}$ photoproduction off the neutron, Phys. Rev. D 92, 094032 (2015).

[18] V. Obraztsov (OKA Collaboration), High statistics measurement of the $K^{+} \rightarrow \pi^{0} e^{+} \nu(\mathrm{Ke} 3)$ decay form factors, Nucl. Part. Phys. Proc. 273-275, 1330 (2016).

[19] B. Velghe (NA62-RK and NA48/2 Collaborations), $K^{ \pm} \rightarrow$ $\pi^{ \pm} \gamma \gamma$ Studies at NA48/2 and NA62-RK experiments at CERN, Nucl. Part. Phys. Proc. 273-275, 2720 (2016).

[20] T. Nagae, The J-PARC project, Nucl. Phys. A805, 486 (2008).

[21] J. He and X. R. Chen, The roles of nucleon resonances in $\Lambda(1520)$ photoproduciton off proton, Phys. Rev. C 86, 035204 (2012).
[22] Y. Huang, J. He, H. F. Zhang, and X. R. Chen, Discovery potential of hidden charm baryon resonances via photoproduction, J. Phys. G 41, 115004 (2014).

[23] J. He, $\Sigma(1385)$ photoproduciton from proton within a Regge-plus-resonance approach, Phys. Rev. C 89, 055204 (2014).

[24] A. Eide et al., Elastic scattering and two-body annihilations at 5 gev/c, Nucl. Phys. B60, 173 (1973).

[25] I. Ambats, D. S. Ayres, R. Diebold, A. F. Greene, S. L. Kramer, A. Lesnik, D. R. Rust, C. E. W. Ward, A. B. Wicklund, and D.D. Yovanovitch, Systematic study of $\pi^{ \pm} p, K^{ \pm} p, p p$, and $\bar{p} p$ forward elastic scattering from 3 to 6 gev/c, Phys. Rev. D 9, 1179 (1974).

[26] C. W. Akerlof et al., Hadron-proton elastic scattering at 50-GeV/c, 100-GeV/c and 200-GeV/c momentum, Phys. Rev. D 14, 2864 (1976).

[27] D. S. Ayres et al. (Fermilab Single Arm Spectrometer Group), $\pi^{ \pm} p, K^{ \pm} p, p p$ and $p \bar{p}$ elastic scattering from 50-GeV/c to 175-GeV/c, Phys. Rev. D 15, 3105 (1977).

[28] M. F. M. Lutz and C. L. Korpa, Open-charm systems in cold nuclear matter, Phys. Lett. B 633, 43 (2006).

[29] J. J. Xie and E. Oset, The $D N, \pi \Sigma_{c}$ interaction in finite volume and the $\Lambda_{c}(2595)$ resonance, Eur. Phys. J. A 48, 146 (2012).

[30] R. S. Azevedo and M. Nielsen, $J / \psi K$ cross-section in meson exchange model, Phys. Rev. C 69, 035201 (2004).

[31] J. He, Understanding spin parity of $P_{c}(4450)$ and $Y(4274)$ in a hadronic molecular state picture, Phys. Rev. D 95, 074004 (2017).

[32] Z. W. Lin and C. M. Ko, A Model for $J / \psi$ absorption in hadronic matter, Phys. Rev. C 62, 034903 (2000).

[33] Y. S. Oh, T. Song, and S. H. Lee, $J / \psi$ absorption by $\pi$ and $\rho$ mesons in meson exchange model with anomalous parity interactions, Phys. Rev. C 63, 034901 (2001).

[34] V. Shklyar, H. Lenske, and U. Mosel, A Coupled-channel analysis of $K \Lambda$ production in the nucleon resonance region, Phys. Rev. C 72, 015210 (2005).

[35] J. Haidenbauer and G. Krein, The Reaction $\bar{p} p \rightarrow \bar{\Lambda}_{c}^{+} \Lambda_{c}^{+}$ close to threshold, Phys. Lett. B 687, 314 (2010).

[36] P. Lebiedowicz and A. Szczurek, $p p \rightarrow p p K^{+} K^{-}$reaction at high energies, Phys. Rev. D 85, 014026 (2012).

[37] T. Mizutani and A. Ramos, $D$ mesons in nuclear matter: A DN coupled-channel equations approach, Phys. Rev. C 74, 065201 (2006).

[38] L. Tolos, A. Ramos, and T. Mizutani, Open charm in nuclear matter at finite temperature, Phys. Rev. C 77, 015207 (2008).

[39] C. Garcia-Recio, V. K. Magas, T. Mizutani, J. Nieves, A. Ramos, L. L. Salcedo, and L. Tolos, The $s$-wave charmed baryon resonances from a coupled-channel approach with heavy quark symmetry, Phys. Rev. D 79, 054004 (2009).

[40] C. Hanhart, Meson production in nucleon-nucleon collisions close to the threshold, Phys. Rep. 397, 155 (2004).

[41] V. Baru, A. M. Gasparyan, J. Haidenbauer, C. Hanhart, A. E. Kudryavtsev, and J. Speth, Production of eta mesons in nucleon-nucleon collisions, Phys. Rev. C 67, 024002 (2003).

[42] M. Tanabashi et al. (Particle Data Group), Review of particle physics, Phys. Rev. D 98, 030001 (2018). 